\title{
Activity From Tidal Disruptions in Galactic Nuclei
}

\author{
Kristen Menou ${ }^{a, 1} \&$ Eliot Quataert ${ }^{b, c, 1}$ \\ ${ }^{a}$ Princeton University, Department of Astrophysical Sciences, Princeton, NJ 08544; \\ kristen@astro.princeton.edu \\ ${ }^{b}$ Institute for Advanced Study, School of Natural Sciences, Einstein Drive, Princeton, NJ 08540 \\ ${ }^{c}$ Present Address: Astronomy Department, UC Berkeley, Berkeley, CA 94720; \\ eliot@astron.berkeley.edu
}

\begin{abstract}
The tidal disruption of a star by a supermassive black hole is expected to lead to a short bright flare followed by an extended period of low-level emission. Existing models of the late-time accretion of the stellar debris via a thin disk imply that the Galactic Center source Sgr A* should be brighter than currently observed by several orders of magnitude. A similar problem exists for M31 and M32. This problem could be avoided if thin disk accretion transitions to low radiative efficiency accretion (e.g., ADAF, CDAF) at low accretion rates (via "evaporation" of the thin disk). Alternatively, we show that the outer portions of a thin disk may become neutral and unable to sustain MHD turbulence on a timescale less than the time between tidal disruption events; this may dramatically shorten the duration of the late accretion phase.
\end{abstract}

subject headings: accretion, accretion disks - black hole physics - MHD - turbulence galaxies: nuclei

\section{Introduction}

A main-sequence star passing within a few Schwarzschild radii of a supermassive black hole $(\mathrm{SMBH})$ of mass $M_{\mathrm{bh}} \lesssim 2 \times 10^{8} \mathrm{M}_{\odot}$ is disrupted by strong tidal forces. Tidal disruption events may therefore offer signatures, in normal (non-active) galaxies, of the presence of a SMBH (e.g. Rees 1990). In recent years, our knowledge of the demographics of SMBHs has improved dramatically, in large part thanks to dynamical studies. There is now strong evidence for a SMBH at the center of our galaxy (Genzel et al. 1997; Ghez et al. 2000) as well as in most galaxies possessing a bulge-component (Magorrian et al. 1998; Gebhardt et al. 2000; Ferrarese \& Merritt 2000; Gebhardt et al. 2001; Merritt et al. 2001). This evidence relies on detailed modeling of the

\footnotetext{
${ }^{1}$ Chandra Fellow
} 
stellar dynamics in galactic nuclei, which also provides reliable estimates of the stellar disruption rate (Magorrian \& Tremaine 1999; Syer \& Ulmer 1999).

Most work on the observational signatures of tidal disruption events has focused on flares (typically in the UV) lasting for a few years and corresponding to the early accretion phase of the stellar debris bound to the SMBH (Rees 1988; Ulmer 1999). Cannizzo, Lee, \& Goodman (1990), however, studied the late time accretion of the stellar debris via a geometrically thin accretion disk. These authors found that the disk accretion rate decreases slowly as a power-law in time, which suggests that remnant activity in galactic nuclei may be visible a long time after the last disruption event occurred. Cannizzo et al. (1990) noted that their results could be problematic in galaxies such as M32, since the nucleus should be brighter than is observed (see also Ulmer 1997).

Here, we revisit the problem of the accretion of stellar debris following a tidal disruption event by a supermassive black hole. In $\S 2$, we briefly review some of the basic theory concerning stellar disruption events. We then point out that the standard accretion scenario, when combined with the latest disruption rates, implies that the SMBH in our Galactic Center (Sgr A*) should be brighter than is observed by several orders of magnitude. In $\S 3$, we reconsider the accretion scenario, paying particular attention to the structure and properties of the accretion flow during both the early super-Eddington phase and the late sub-Eddington phase. In $\S 4$, we conclude with a few possible extensions of this work.

\section{Basics of Tidal Disruption}

\subsection{Theory}

A solar-type star is tidally disrupted if its orbit brings it closer to a SMBH (with $\left.M_{\mathrm{bh}} \lesssim 2 \times 10^{8} \mathrm{M}_{\odot}\right)$ than the tidal radius $R_{\mathrm{T}} \approx 5 M_{7}^{-2 / 3} R_{s}$, where $M_{7}=M_{\mathrm{bh}} / 10^{7} M_{\odot}$ and $R_{s}=3 \times 10^{12} M_{7} \mathrm{~cm}$ is the Schwarzschild radius of the SMBH (e.g. Hills 1975). Following the disruption, about half of the stellar debris is bound to the SMBH and half is unbound. The bound material is nearly uniformly distributed in binding energy and so is distributed as $P^{-5 / 3}$ in orbital period, with a minimum orbital period $P_{\min } \approx 0.7 M_{7}^{1 / 2}$ yr (Rees 1988; Evans \& Kochanek 1989).

The subsequent evolution of the bound stellar debris is both difficult to predict analytically and to follow numerically (Lacy, Townes \& Hollenbach 1982; Rees 1988; Evans \& Kochanek 1989; Cannizzo et al. 1990; Kochanek 1994). It is unclear what fraction of the initially bound material remains bound after the second or subsequent periastron passages (violent shocks and significant gas heating being expected from stream-stream collisions). The latest numerical simulations indicate that about $25 \%$ of the initial stellar mass may remain bound after the second periastron passage (Ayal, Livio \& Piran 2000).

Although the process of circularization by stream-stream collisions is complex, one expects circularization to occur on a timescale corresponding to at most a few orbital timescales, $P_{\min }$, at 
pericenter (Ulmer 1999). Given the distribution of binding energy of the bound material, mass

returns to pericenter at a rate $\dot{M} \approx M_{7}^{-1 / 2}\left(t / P_{\min }\right)^{-5 / 3} M_{\odot} \mathrm{yr}^{-1}$ for $t \gtrsim P_{\min }$ (Rees 1988; Phinney 1989). This is larger than the Eddington accretion rate $\approx 0.1 M_{7} M_{\odot} \mathrm{yr}^{-1}$ for several years and so accretion should initially proceed via a photon trapped radiatively inefficient accretion flow (Rees 1988; Ulmer 1997; see §3). The viscous timescale in such a hot flow is indeed short enough for the material to accrete as fast as it circularizes (unless the viscosity parameter $\alpha$ is $\ll 0.1$; Ulmer 1999).

After a few years, the infall rate drops below the Eddington rate, photons are no longer trapped in the accretion flow, and the gas begins to cool efficiently. Mass returning to pericenter then begins to accrete via a geometrically thin disk, with the accretion rate set by the long viscous time in the thin disk, not the rate at which mass returns to pericenter. Cannizzo et al. (1990) studied the thin disk accretion phase using numerical simulations and analytic self-similar solutions. They assumed that the accretion disk has nearly constant total angular momentum; viscosity can redistribute angular momentum (from material at small radii to material at large radii), but it is assumed that there is no global loss of angular momentum from the system such as would be provided by a jet or an MHD wind. Under these assumptions, Cannizzo et al. found that the accretion rate onto the SMBH decays as a weak power-law in time: $\dot{M} \propto t^{-n}$, with $n \simeq 1.2$ for various opacity laws and almost independent of the value of the viscosity parameter $\alpha$ in the disk. As the disk mass decreases due to accretion, the mean radius of the disk increases in order to conserve angular momentum (roughly as $R(t) \propto t^{3 / 8}$ ).

\subsection{Observational Constraints}

Attempts to identify tidal disruption events have mostly focused on detecting the initial "flare" of emission with a timescale of $\approx P_{\min } \approx$ years; these are short-lived events with a very low duty-cycle per galaxy of $\sim 10^{-4}$ (e.g. Rees 1988; Loeb \& Ulmer 1997; Ulmer, Paczynski \& Goodman 1998; Komossa \& Dahlem 2001). Another approach to the problem is to look for the late emission from tidal disruption events, as expected from the study of disk accretion by Cannizzo et al. (1990). In this scenario, the issue is not one of short duration, but rather of the detectability of low-level remnant activity in a galactic nucleus. To address this question quantitatively, we searched the literature for a set of nearby galaxies meeting the following three criteria: (1) estimates of tidal disruption rates based on recent dynamical studies are available (Magorrian \& Tremaine 1999; Syer \& Ulmer 1999); (2) the estimated SMBH mass is below $2 \times 10^{8} M_{\odot}$, the maximum mass allowed for main-sequence stellar disruption; (3) detections of, or useful upper limits on, low-level nuclear activity are available. The three galaxies that met these criteria are our own Galaxy (i.e. Sgr A*), M31 and M32.

Table 11 summarizes the relevant data for these three galaxies. For the Galactic Center main-sequence disruption rate, we adopt $10^{-4} \mathrm{yr}^{-1}$, which is somewhat in excess of the value quoted by Syer \& Ulmer (1999). This is because these authors tend to underestimate the rates as 
compared to Magorrian \& Tremaine (1999).2 For the bolometric luminosities, we conservatively adopt the highest luminosity value available for each nucleus among the various observed spectral bands (when detected).

For thin disk accretion, the luminosity limits in Table 1 can be converted into accretion rate limits for a radiative efficiency $\approx 10 \%$. We find that the accretion rates onto the SMBHs in Sgr $\mathrm{A}^{*}, \mathrm{M} 31, \& \mathrm{M} 32$ would be $\approx 10^{16},<10^{17}$ and $<5 \times 10^{16} \mathrm{~g} \mathrm{~s}^{-1}$, respectively, for radiatively efficient accretion. These accretion rates are very small in the context of tidal disruption theory: for $\alpha=0.1$ and an initial $\approx 0.1 M_{\odot}$ disk of tidally disrupted material (see $\S 3$ ), Cannizzo et al.'s (1990) thin disk models predict that the accretion rate onto the SMBH drops below $10^{19} \mathrm{~g} \mathrm{~s}^{-1}$ approximately $4 \times 10^{4}$ yrs following a tidal disruption event (see their Fig. 3b), while it reaches values $\leq 10^{17} \mathrm{~g} \mathrm{~s}^{-1}$ only well after $10^{6}$ years. We expect these predictions to be relatively robust given the very weak dependence of $\dot{M}(t)$ on viscosity and opacity. Since $10^{6}$ years is much longer than the expected time between two consecutive stellar disruptions, disk accretion of stellar debris as described by Cannizzo et al. (1990) predicts that Sgr A*, M31, and M32 should be continuously brighter than is presently observed. This is illustrated in Table 1, where we also list, for the three galactic nucei of interest, the luminosity expected in Cannizzo et al.'s thin disk models after a time corresponding to the interval between successive disruption events. Note that our analysis is conservative since we do not consider the emission spectrum expected from thin accretion disks (expected to be prominent in the IR-UV, where the observational limits are generally stronger than our adopted bolometric luminosities).

\section{Revisions to the Accretion Model for Tidal Disruption Events}

One possible resolution of this observational puzzle is the following: for accretion rates below $\approx 1 \%$ of the Eddington accretion rate, i.e., $\lesssim 10^{-3} M_{7} M_{\odot} \mathrm{yr}^{-1}$, gas can accrete onto a black hole via a hot optically thin low radiative efficiency accretion flow (LRAF) such as an advection-dominated accretion flow (ADAF; Rees et al. 1982; Abramowicz et al. 1995; Narayan \& Yi 1994; 1995) or its variants, ADIOSs (Blandford \& Begelman 1999) and CDAFs (Quataert \& Gruzinov 2000; Narayan et al. 2000). Typically, an accretion rate $\dot{M} \approx 10^{-2} \dot{M}_{\text {Edd }}$ is reached years to decades after a tidal disruption event. If thin disk accretion transitions to LRAF accretion at this time, the accretion luminosity will precipitously drop, either because the radiative efficiency decreases dramatically (ADAFs) or because the accretion rate decreases dramatically (CDAFs/ADIOS), or perhaps both. This scenario may provide an explanation for the absence of late time emission from the accretion of tidally disrupted stars (see also Ulmer 1997).

However, the thin disk $\rightarrow$ LRAF model requires positing that thin disks spontaneously "evaporate" into LRAFs when $\dot{M} \lesssim 10^{-2} \dot{M}_{\text {Edd }}$ (thin disks remain, after all, a viable mode of

\footnotetext{
${ }^{2}$ Presumably, this is due to the additional stellar orbits considered by Magorrian \& Tremaine in their calculation.
} 
accretion even for $\left.\dot{M} \ll \dot{M}_{\text {edd }}\right)$. This possibility has been extensively and successfully applied to observations of time-dependent accretion flows in X-ray binaries (e.g., Esin et al. 1997; 1998; 2001), but the "evaporation" process - and hence its dependence on, e.g., black hole mass - is not well understood. It therefore seems worthwhile exploring scenarios in which accretion continues to proceed via a thin disk even for $\dot{M} \ll \dot{M}_{\text {Edd }}$.

\subsection{Early Super-Eddington Phase}

The initial accretion phase of tidal disruption events are likely to proceed via a superEddington accretion flow in which the radiative efficiency is low because photons are trapped in the accretion flow (see $\S 2.1$ ). Recent work on such LRAFs indicates that much of the mass in the accretion disk may be lost to an outflow. Blandford \& Begelman (1999) proposed this based on analytic arguments (see also Narayan \& Yi 1994). In addition, numerical simulations of LRAFs have found that the accretion flow structure adjusts so as to transport a significant flux of energy $\left(\sim 0.01 \dot{M} c^{2}\right)$ from small to large radii (e.g., Stone, Pringle, \& Begelman 1999; Igumenshchev \& Abramowicz 1999, 2000); this is the CDAF regime described analytically by Quataert \& Gruzinov (2000) and Narayan et al. (2000). Although the fate of this outward energy flux is not specified in either the analytic calculations or the numerical simulations, it seems very likely that this energy drives a strong outflow from the outer edge of the accretion flow (see also Hawley, Balbus \& Stone 2001).

The initial super-Eddington phase is important in the context of tidally disrupted stars because it sets the initial conditions for the subsequent thin disk evolution. In particular, rather than having the entire bound stellar debris available to accrete, we expect most of this to be blown away in a strong outflow. Only the material that returns to pericenter at significantly sub-Eddington rates remains bound to the black hole. Assuming that $0.25 \mathrm{M}_{\odot}$ of debris remains bound to the hole after second periastron passage and that essentially all the mass that would be accreted during the super-Eddington phase is instead lost to infinity, we deduce from $\S 2.1$ that the mass of bound material left to accrete at sub-Eddington rates is $\lesssim 0.1 M_{7}^{3 / 5} M_{\odot}$.

\subsection{Late Sub-Eddington Phase}

For the $\sim 0.1 M_{\odot}$ accreted at a sub-Eddington rate, the flow will adopt a thin disk configuration and the solution described by Cannizzo et al. (1990) should initially apply. As the accretion disk spreads and cools down, however, the gas eventually reaches temperatures $\lesssim 10^{4} \mathrm{~K}$ for which the disk is subject to the thermal ionization instability caused by hydrogen recombination (Meyer \& Meyer-Hofmeister 1981; Lin \& Shields 1986; Cannizzo 1992; Siemiginovska, Czerny \& Kostyunin 1996). Following Menou \& Quataert (2001), we estimate that the effect of the ionization instability should be limited to the outermost regions of tidal debris disks and will not 
lead to substantial changes in the disk's global evolution. As the disk spreads somewhat further, however, it may eventually become so cool $(\sim 1000-2000 \mathrm{~K})$ and neutral that MHD turbulence cannot be sustained.

MHD turbulence and gravitational instability are presently the only robust candidates for angular momentum transport in isolated accretion disks (e.g., Balbus \& Hawley 1998; Gammie 2001). Since tidal debris disks have very low masses they are probably gravitationally stable. If the outermost regions of a tidal debris disk become passive when MHD turbulence ceases to operate, the disk's viscous evolution will be strongly modified. Active accretion will proceed only in regions of the disk with $T_{c} \gtrsim 1000 \mathrm{~K}$, which are confined to smaller and smaller radii as $\dot{M}$ decreases with time.

To quantitatively estimate when the disk's viscous evolution is modified, we note that a disk annulus becomes thermally unstable when the accretion rate locally drops below the value $\dot{M}_{\text {crit }}(R) \simeq 6 \times 10^{17} M_{7}^{-0.9} R_{13}^{2.7} \mathrm{~g} \mathrm{~s}^{-1}$, where $R_{13}$ is the radius of interest in units of $10^{13} \mathrm{~cm}$ (see, e.g., Hameury et al. 1998). After an initial transient phase of duration $t_{0}$ (tens of years, set by the viscous time in the thin disk), the disk accretion rate and the mean radius of the disk evolve roughly as $\dot{M} \propto t^{-1.2}$ and $R(t) \propto t^{3 / 8}$, respectively ( $\$ 2.1$ ), so that $\dot{M} / R^{2.7} \propto t^{-2.2}$. Combining this scaling with the one for the critical rate, $\dot{M}_{\text {crit }}$, we find that the disk's outer regions become subject to the thermal ionization instability at a time

$$
t_{n}=t_{0}\left[\frac{\dot{M}\left(t_{0}\right)}{6 \times 10^{17} \mathrm{~g} \mathrm{~s}^{-1}} \frac{M_{7}^{0.9}}{R_{13}^{2.7}\left(t_{0}\right)}\right]^{\frac{1}{2.2}},
$$

where $\dot{M}\left(t_{0}\right)$ and $R_{13}\left(t_{0}\right)$ are the values of the disk accretion rate and disk outer radius at time $t_{0}$, respectively.

For an initial disk mass of $M_{d} \approx 0.1 \mathrm{M}_{\odot}, \dot{M}\left(t_{0}\right) \sim M_{d} / t_{0}$, since a reasonable fraction of the disk's mass is accreted in the initial few viscous times. Moreover, the initial outer radius of the disk is of order the tidal disruption radius, i.e., $R\left(t_{0}\right) \approx R_{T} \approx 2 \times 10^{13} M_{7}^{1 / 3} \mathrm{~cm}$. Thus,

$$
t_{n} \approx 3 \times 10^{3}\left(\frac{t_{0}}{10 \mathrm{yrs}}\right)^{0.55}\left(\frac{M_{d}}{0.1 M_{\odot}}\right)^{0.45}\left(\frac{R\left(t_{0}\right)}{R_{T}}\right)^{-1.2} \text { years. }
$$

For $R\left(t_{0}\right)$ closer to $10^{14} \mathrm{~cm}$, as suggested by Cannizzo et al.'s simulations (because the disk spreads away from the tidal disruption radius during the transient accretion phase, $\left.t_{0}\right)$, the above estimate for $t_{n}$ is reduced by a factor of $\approx 5$, to $\lesssim 10^{3}$ years. This is smaller than the typical time between tidal disruption events in our Galaxy, M31 and M32, implying that a single power law will not adequately describe the late time evolution of tidal debris disks in galactic nuclei.

Lipunova \& Shakura (2000) have shown that thin $\alpha$-disks with fixed outer radii evolve according to $\dot{M} \propto t^{-3.3}$ and $\dot{M} \propto t^{-2.5}$ for free-free and electron scattering opacity, respectively. The evolution is much faster than a disk which conserves angular momentum $\left(\dot{M} \propto t^{-1.2}\right)$ because the disk is not forced to spread to larger radii where the viscous time is longer. In our problem, 
the radius bounding the active region shrinks with time and so the accretion rate will decrease even more rapidly for $t>t_{n}$.

These results imply that once the outer regions of the disk become cold enough, the accretion rate and accretion luminosity from remnant tidal debris disks will precipitously drop. It is unclear, however, whether this is quantitatively sufficient to account for the observational constraints in §2.1. In particular, assuming the sharp drop in $\dot{M}(t)$ happens a time $\sim t_{n}$ after a stellar disruption, the disk accretion rate still has to decrease by $3-4$ orders of magnitude from $\dot{M}\left(t_{n}\right) \sim 10^{20} \mathrm{~g} \mathrm{~s}^{-1}$ to satisfy the limits on bolometric luminosities given in Table 1 (disk accretion being radiatively efficient). Given that the ratio of the time between two consecutive disruption events and $t_{n}$ is as small as $\sim 10$ for the three galaxies of interest, this implies that a power law evolution of the disk accretion rate steeper than $t^{-4}$ is required to just satisfy the observational limits. If one further requires these nuclei to be as dim as currently observed for most of the time between consecutive disruption events, the power law has to be even steeper than this.

\section{Conclusion}

We have reconsidered the accretion of stellar debris following tidal disruption by a supermassive black hole. We argued that a large fraction of the mass initially bound to the black hole will be lost to infinity via an outflow during the early super-Eddington accretion phase. This limits the mass available for late time accretion via a thin disk to $\sim 0.1 M_{\odot}$. We also suggest that, on a timescale of $\sim 10^{3}$ years, the thin disk accretion rate will decrease much more quickly than in the standard similarity solution of Cannizzo et al. (1990) - this is because the disk has spread so far from the black hole that its outer regions are cold, mostly neutral, and may be unable to sustain MHD turbulence. Quantitatively, however, it is unclear if these two effects can explain the very low level of activity observed in the nuclei of our Galaxy, M31 and M32.

There are a number of uncertain elements in this evolutionary scenario that require clarification before a firm statement can be made on the level of activity expected after tidal disruptions in galactic nuclei. Perhaps paramount among them is whether thin disks evaporate into low-radiative efficiency accretion flows (LRAFs) once the accretion rate is sufficiently sub-Eddington. For purely thin disk accretion scenarios, a detailed numerical investigation of the evolution of a disk with an actively accreting zone that shrinks with time, as described in $\S 3.2$, would be particularly useful. Note that there are also additional effects that could further reduce the level of activity expected, such as significant angular momentum losses to a jet or an MHD wind during the evolution. 


\section{Acknowledgments}

Support for this work was provided by NASA through Chandra Fellowship grant PF9-10006 (to KM) and PF9-10008 (to EQ) awarded by the Smithsonian Astrophysical Observatory for NASA under contract NAS8-39073. KM thanks the Center for Astrophysical Sciences at Johns Hopkins University for hospitality.

\section{REFERENCES}

Abramowicz, M.A., Chen, X., Kato, S., Lasota, J.-P. \& Regev, O. 1995, ApJ, 438, L37

Ayal, S., Livio, M. \& Piran, T. 2000, ApJ 545, 772

Baganoff, F. K., et al. 2000, AAS HEAD meeting, 32, 02.10.

Balbus, S.A., \& Hawley, J.F. 1998, Rev. Mod. Phys., 70, 1

Blandford, R.D. \& Begelman, M.C. 1999, MNRAS, 303, L1

Cannizzo J.K., 1992, ApJ, 385, 94

Cannizzo, J.K., Lee, H. M. \& Goodman, J. 1990, ApJ, 351, 38

Esin, A.A., McClintock, J.E. \& Narayan, R. 1997, ApJ, 489, 865

Esin, A.A., Narayan, R., Cui, W., Grove, J.E. \& Zhang, S.-N. 1998, ApJ, 505, 854

Esin, A. A. et al., 2001, ApJ, 555, 483

Eskridge, P.B., White, R.E., III \& Davis, D.S. 1996, ApJ, 463, L59

Evans, C.R. \& Kochanek, C.S. 1989, ApJ, 346, L13

Ferrarese, L. \& Merritt, D. 2000, ApJ, 539, L9

Gammie, C. F. 2001, ApJ, 553, 174

Garcia, M.R. et al. 2001, astroph/0012387

Gebhardt, K. et al. 2000, ApJ, 539, L13

Gebhardt, K. et al. 2001, AJ, in press (astroph/0107135)

Genzel, R., Eckart, A., Ott, T. \& Eisenhauer, F. 1997, MNRAS, 291, 219

Ghez, A. M., Morris, M., Becklin, E. E., Tanner, A. \& Kremenek, T. 2000, Nature, 407, 349

Gregory, P.C. \& Condon, J.J. 1991, ApJS, 75, 1011 
Hameury J.-M., Menou K., Dubus G., Lasota J.-P., Huré J.-M. 1998, MNRAS, 298, 1048

Hawley, J.F., Balbus, S.A. \& Stone, J.M. 2001, ApJ, 554, L49

Hills, J.G. 1975, Nature, 254, 295

Igumenshchev, I.V. \& Abramowicz, M.A. 1999, MNRAS, 303, 309

Igumenshchev, I.V. \& Abramowicz, M.A. 2000, ApJS, 130, 463

Knapp, G.R., Guhathakurta, P., Kim, D.-W. \& Jura, M.A. 1989, ApJS, 70, 329

Kochanek, C.S. 1994, ApJ, 422, 508

Komossa, S. \& Dahlem, M. 2001, astroph/0106422

Lacy, J.H., Townes, C.H. \& Hollenbach, D.J. 1982, ApJ, 262, 120

Lin, D.N.C. \& Shields, G.A. 1986, ApJ, 305, 28

Lipunova, G. V. \& Shakura, N. I. 2000, A\&A, 356, 363

Loeb, A. \& Ulmer, A. 1997, ApJ, 489, 573

Loewenstein, M., Hayashida, K., Toneri, T. \& Davis, D.S. 1998, ApJ, 497, 681

Magorrian, J., Tremaine, S. et al. 1998, AJ, 115, 228

Magorrian, J. \& Tremaine, S. 1999, MNRAS, 309, 447

Menou, K. \& Quataert, E. 2001, ApJ, 552, 204

Merrit, D., Ferrarese, L. \& Joseph, C.L. 2001, Science, in press (astroph/0107359)

Meyer F. \& Meyer-Hofmeister E. 1981, A\&A, 104, L10

Narayan, R., Igumenshchev, I.V. \& Abramowicz, M.A. 2000, ApJ, 539, 798

Narayan, R., Mahadevan, R., Grindlay, J.E., Popham, R.G. \& Gammie, C. 1998, ApJ, 492, 554

Narayan, R. \& Yi, I. 1994, ApJ, 428, L13

Narayan, R. \& Yi, I. 1995, ApJ, 452, 710

Phinney, E.S. 1989, in IAU Symp. 136, The Galactic Center, Ed. M. Morris (Dordrecht: Kluwer)

Quataert, E. \& Gruzinov, A. 2000, ApJ, 539, 809

Rees, M.J. 1988, Nature, 333, 523

Rees, M.J. 1990, Science, 247, 817 
Rees, M.J., Phinney, E.S., Begelman, M.C. \& Blandford, R.D. 1982, Nature, 295, 17

Shakura, N.I. \& Sunyaev, R.A. 1973, A\&A, 24, 337

Siemiginowska, A., Czerny, B. \& Kostyunin, V., 1996, ApJ, 458, 491

Stone, J.M., Pringle, J.E. \& Begelman, M.C. 1999, MNRAS, 310, 1002

Syer, D. \& Ulmer, A. 1999, MNRAS, 306, 35

Ulmer, A. 1997, astroph/9708265

Ulmer, A. 1999, ApJ, 514, 180

Ulmer, A., Paczynski, B. \& Goodman, J. 1998, A\&A, 333, 379 
Table 1: NEARBY GALACTIC NUCLEI

\begin{tabular}{ccccc}
\hline \hline & $\begin{array}{c}\text { Black Hole Mass } \\
\left(\mathrm{M}_{\odot}\right)\end{array}$ & $\begin{array}{c}\text { Stellar Disruption Rate } \\
\left(\mathrm{yr}^{-1}\right)\end{array}$ & $\begin{array}{c}\text { Bolometric Luminosity } \\
\left(\mathrm{erg} \mathrm{s}^{-1}\right)\end{array}$ & $\begin{array}{c}\text { Expected Luminosity } \\
\left(\operatorname{erg~s}^{-1}\right)\end{array}$ \\
\hline Milky Way & $2.6 \times 10^{6} \mathrm{~b}$ & $10^{-4 c}$ & $10^{36 e}$ & $\sim 5 \times 10^{39}$ \\
M31 & $3.5 \times 10^{7} \mathrm{~b}$ & $10^{-4.68 d}$ & $<10^{37 \mathrm{f}}$ & $\sim 10^{39}$ \\
M32 & $3.7 \times 10^{6} \mathrm{~b}$ & $10^{-3.62 d}$ & $<5 \times 10^{36 \mathrm{~g}}$ & $\sim 10^{40}$ \\
\hline
\end{tabular}

NOTE. - (a) Adapted from Cannizzo et al. (1990) (b) Gebhardt et al. (2000) (c) Adapted from Syer \& Ulmer (1999) (d) Magorrian \& Tremaine (1999) (e) Broadband: Narayan et al. (1998); X-ray: Baganoff et al. (2001) (f) Radio: Gregory \& Condon (1991); X-ray: Garcia et al. (2001) (g) Far-IR: Knapp et al. (1989); Steady X-ray: Eskridge, White \& Davis (1996), Loewenstein et al. (1998). 